\title{
RUNOFF VARIABILITY IN THE SCOTT RIVER (SW SPITSBERGEN) IN SUMMER SEASONS 2012-2013 IN COMPARISON WITH THE PERIOD 1986-2009
}

\author{
Łukasz FranczaK, Waldemar Kociuba, GrZegorz GajeK \\ Faculty of Earth Sciences and Spatial Management, Maria Curie-Skłodowska University in Lublin, Poland
}

Manuscript received: March 8, 2016

Revised version: June 21, 2016

\begin{abstract}
FranczaK Ł., Kociuba W., GajeK G., 2016. Runoff variability in the Scott River (SW Spitsbergen) in summer seasons 2012-2013 in comparison with the period 1986-2009. Quaestiones Geographicae 35(3), Bogucki Wydawnictwo Naukowe, Poznań, pp. 39-50, 7 figs, 1 table.
\end{abstract}

\begin{abstract}
River runoff variability in the Scott River catchment in the summer seasons 2012 and 2013 has been presented in comparison to the multiannual river runoff in 1986-2009. Both in particular seasons and in the analysed multiannual, high variability of discharge rate was recorded. In the research periods 2012-2013, a total of 11952 water stages and 20 flow rates were measured in the analysed cross-section for the determination of 83 daylong discharges. The mean multiannual discharge of the Scott River amounted to $0.96 \mathrm{~m}^{3} \mathrm{~s}^{-1}$. The value corresponds to a specific runoff of 94.6 $\mathrm{dm}^{3} \cdot \mathrm{s}^{-1} \mathrm{~km}^{2}$, and the runoff layer $937 \mathrm{~mm}$. The maximum values of daily discharge amounted to $5.07 \mathrm{~m}^{3} \cdot \mathrm{s}^{-1}$, and the minimum values to $0.002 \mathrm{~m}^{3} \cdot \mathrm{s}^{-1}$. The highest runoff occurs in the second and third decade of July, and in the first and second decade of August. The regime of the river is determined by a group of factors, and particularly meteorological conditions affecting the intensity of ablation, and consequently river runoff volume. We found a significant correlation (0.60 in 2012 and 0.67 in 2013) between the air temperature and the Scott River discharge related to the Scott Glacier ice melt.
\end{abstract}

KEY WORDS: hydrology, proglacial river, polar region, Svalbard

Corresponding author: Łukasz Franczak (e-mail: lukasz.franczak@poczta.umcs.lublin.pl)

\section{Introduction}

The runoff regime of a river is determined by climatic and relief conditions. The primary source of water supply to rivers with glacial regime is glacial ablation, strictly dependent on the thermal conditions occurring in a given period (Krenke and Khodakov 1966, Baranowski and Głowicki 1975, Piasecki and Pulina 1975, Collins 1979, Leszkiewicz 1987, Pereyma 1991, Bartoszewski 1998, Brykała and Araźny 2002, Sobota 2004, 2013, Bartoszewski et al. 2009). The river regime in glacial catchments is particularly determined by the degree of catchment glaciation, size of the glacier, and hydrological processes occurring within the glacier, related to the level of development of the drainage system determining the routes of circulation of supraglacial, inglacial, and subglacial waters (Baranowski 1977, Bartoszewski 1998, Sobota 2014). Factors modifying river runoff include land relief and catchment lithology (Sobota 2013, Kociuba and Janicki 2015a, b).

The river runoff in polar regions in the summer is dominated by proglacial water, with a lower contribution of pronival water related to melting of snow patches, atmospheric precipitation, 
and seasonal thaw of active layer in permafrost (Leszkiewicz 1987, Marciniak and Marszelewski 1990, Tranter et al. 1996, Bartoszewski 1998, Sobota 2013). It is distinctive of glacial rivers that more than $80 \%$ of the value of annual runoff corresponds to the spring and summer period (Kuziemski 1959, Østrem 1973, Leszkiewicz 1982, Bartoszewski 1998, Rachlewicz 2007). In the winter season runoff occurs in the form of episodic outflows of englacial and subglacial channels (Stenborg 1965, Grześ and Sobota 2000, Hodgkins et al. 2004, Sobota 2011).

Rivers with glacial regime are distinguished by high runoff variability corresponding with changes in the rate of glacial ablation. It is manifested in the daily and seasonal rhythm, and non-periodical and multiannual runoff variability (Klæboe 1948, Leszkiewicz 1987, Tranter et al. 1996, Bartoszewski 1998, Sobota 2013).

The river runoff variability in Spitsbergen has been a subject of a number of publications (Piasecki and Pulina 1975, Głowicki 1982, Bartoszewski 1987, 1998, Hodgkins 1997, Sobota 1998, 2004, 2013, Hagen et al. 2000, Rachlewicz
2007, Bartoszewski et al. 2009), although a considerable part of them is based on observations considering a single measurement period. Analyses concerning longer, multiannual periods are encountered in the literature more seldom (Bartoszewski 1998, 2007, Killingtveit et al. 2003, Sobota 2013, 2014).

The aim of the study is to analyse the runoff variability of the Scott River in summer seasons 2012-2013, and to compare it with the results of research conducted in the period 1986-2009.

\section{Study area}

The Scott River catchment is located in the SW part of the Wedel-Jarlsberg Land (SW Spitsbergen), flowing out of a glacier of the same name (Fig. 1 and 2).

The upper part of the catchment, covering the partially glaciated montane valley, is built of rocks of the Upper Proterozoic developing the Hecla-Hoek formation, and postglacial Quaternary formations. The lower part

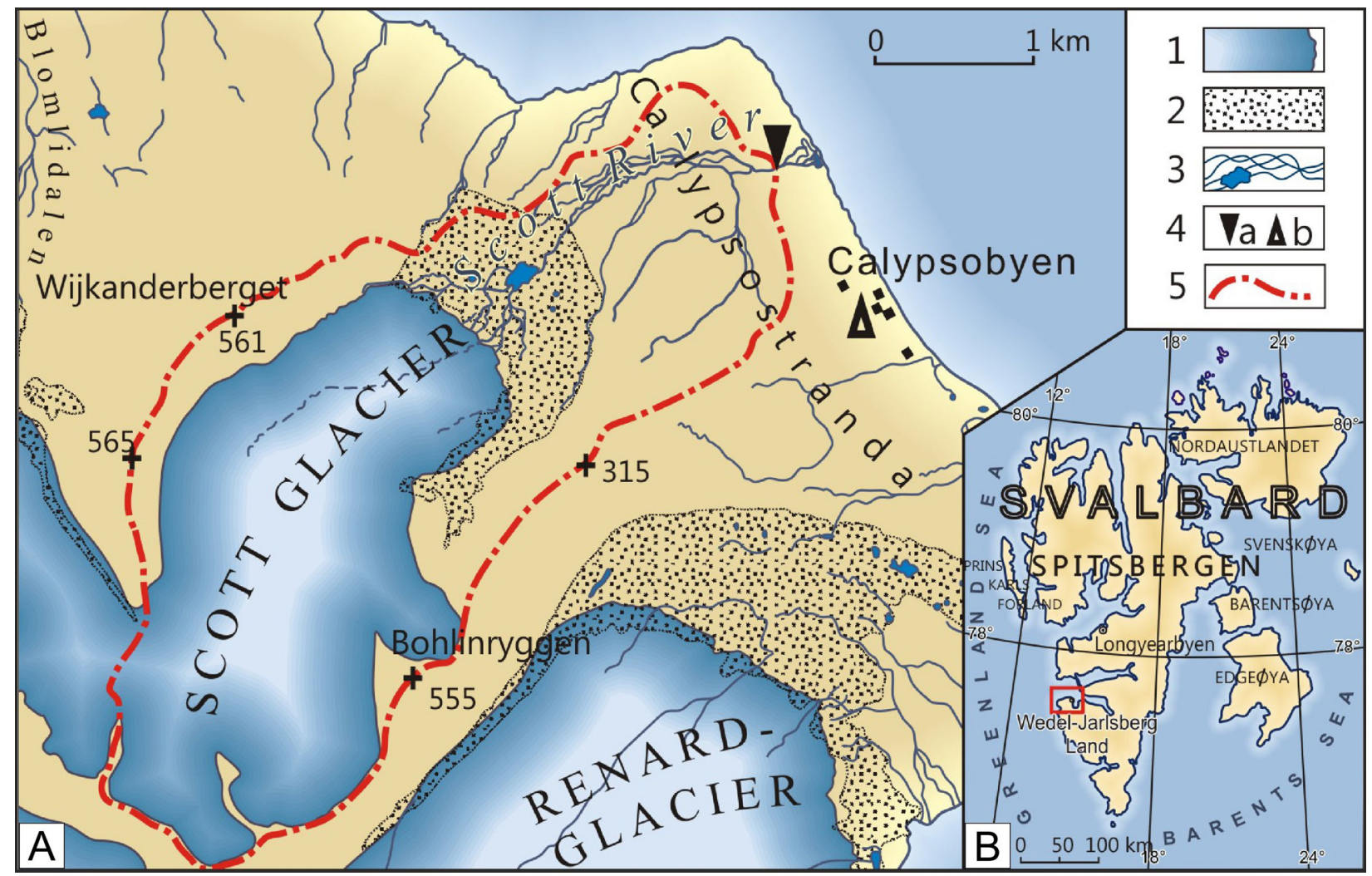

Fig. 1. A - Location of the study area: 1 - valley glaciers, 2 - glacial accumulation zones, 3 - rivers and water bodies, $4 \mathrm{a}$ - water gauge station; $4 \mathrm{~b}$ - meteorological station, 5 - border of the Scott River catchment. B - Location of the study area on Svalbard Archipelago. 


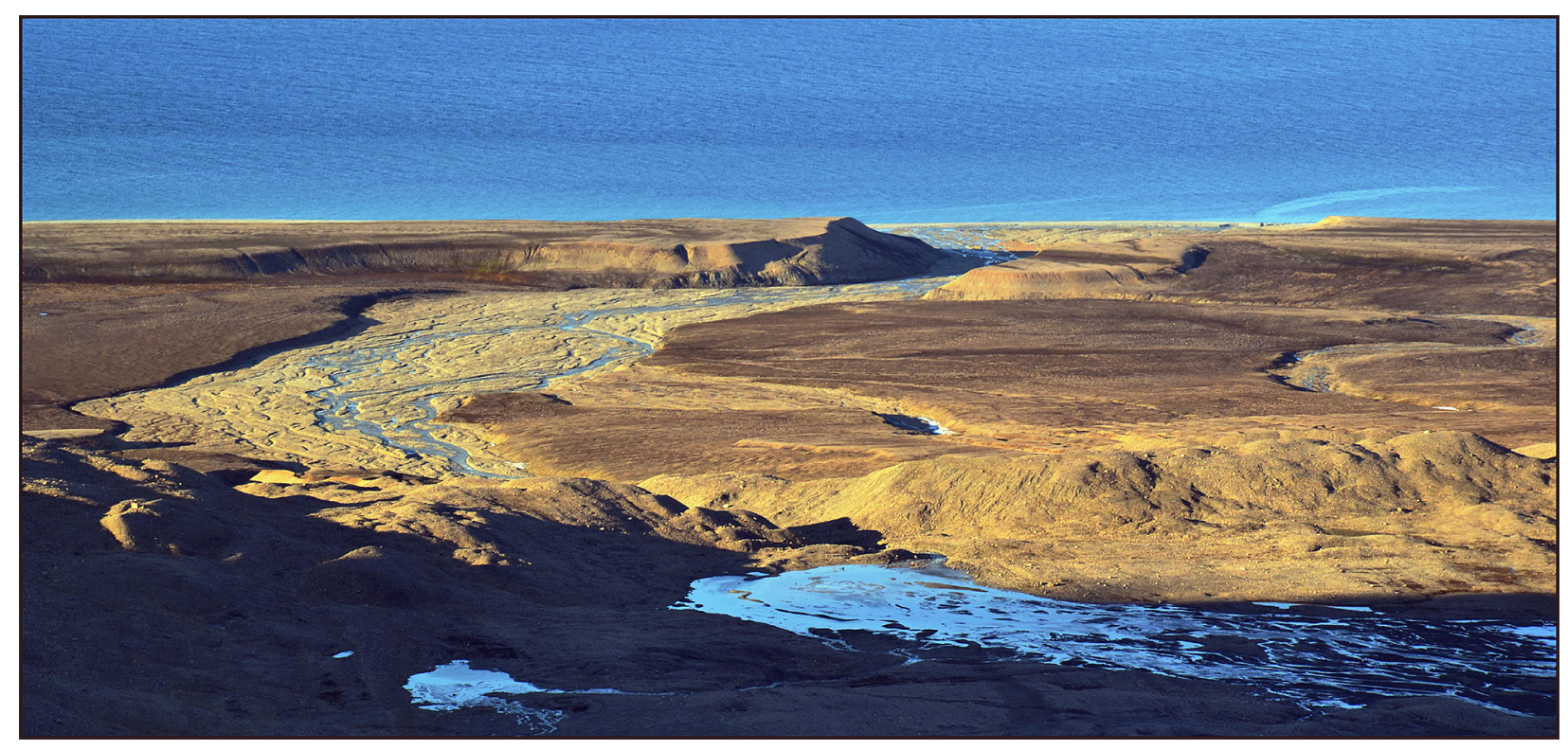

Fig. 2. Downstream view of the central and lower part of the Scott River valley with variable channel patterns.

- Calypsostranda - is a seashore plain composed of uneven-aged marine terraces built of Tertiary and Quaternary sediments (Flood et al. 1971, Pękala 1987, Dallmann et al. 1990).

The catchment area of the Scott River is 10.1 $\mathrm{km}^{2}$ and the glacier occupied approximately 4.0 $\mathrm{km}^{2}$ in the years 2012-2013 (40\% of the total catchment area). The Scott Glacier is a valley glacier of alpine type showing strong ice mass loss and quick glacier retreat since the 1930s (Pillewizer 1939). In the study period (2012), the glacier's terminus reached an altitude of $85 \mathrm{~m}$ a.s.l., and its highest parts up to $502 \mathrm{~m}$ a.s.l. The average altitude of the catchment is $267 \mathrm{~m}$ a.s.l. The length of the glacier amounts to $3.1 \mathrm{~km}$. Its width varies from 1.1 to $1.8 \mathrm{~km}$, and the mean inclination of the longer axis is $0.23 \mathrm{~m} \cdot \mathrm{m}^{-1}$ (Kociuba and Janicki $2015 b)$. The glacier is of NW-SE orientation. It is confined to the mountain range Bohlinryggen to SW, and Wijkanderberget to NW.

According to the Pardè classification (1957) the Scott River represents glacial regime. The glacial drainage system is composed of subglacial, englacial, and supraglacial channels of various sizes. After leaving the glacier, they are divided into numerous channels distributing waters to the intramarginal outwash plain. Then, through a gorge in the terminal moraine rampart, the waters are flow in a single compact channel to the area of the extramarginal outwash plain, developing an extensive braided system (Kociuba and Janicki 2015b, Kociuba et al. 2015). In its lower course, the river developed a gorge dissecting the system of elevated marine terraces. Below, it developed an alluvial fan separated from the fjord with a storm rampart, dissected in its south-western part by the water outflow to the Recherchefjorden. The length of the primary river channel with the biggest discharge amounts to $3.3 \mathrm{~km}$. It begins at the elevation of $92.5 \mathrm{~m}$ a.s.l. The mean slope inclination of the glacier-free part of the catchment amounts to $0.028 \mathrm{~m} \cdot \mathrm{m}^{-1}$ (Kociuba et al. 2014, Kociuba and Janicki 2015b, Fig. 1 and 2).

\section{Methods}

The analysis of the runoff variability of the Scott River applied the results of own measurements conducted in periods from 12.07 to 23.08.2012 and from 10.07 to 18.08.2013, as well as data gained in publications of other authors (Bartoszewski 1987, 1988, 1989, 1991, 1998, 2007, Michalczyk 1990, Bartoszewski et al. 2009, Chmiel et al. 2012, Kociuba et al. 2014). Their research concerned to the Scott River hydrology in the period 1986-2009.

The Scott River discharge was measured in 2012 and 2013 in the water level gauge located approximately $350 \mathrm{~m}$ above the river mouth to the Recherchefjorden. The measurement point is located in the gorge cutting through the marine terraces hence almost all waters from the 
catchment met the water level gauge (Fig. 1). A pressure water level logger (Schlumberger Water Services) with measurement accuracy of $0.5 \mathrm{~cm}$ was installed in the Scott River channel, recording changes in the water level and water temperature at a time resolution of 10 minutes. River runoff was determined based on the rating curve equation developed based on periodical measurements of river discharge. The river discharge was measured twice a week with a current meter Hega II and Acoustic Digital Current meter (OTT ADC).

Simultaneously with hydrological research, meteorological measurements were conducted including: air temperature, atmospheric precipitation, and wind velocity and direction. The measurements were performed every 10 minutes by means of an automatic meteorological station (Campbell Scientific CR10). Dataloger was located at an elevated marine terrace near the Calypso settlement, at an altitude of $23 \mathrm{~m}$ a.s.l. at a distance of approximately $1 \mathrm{~km}$ from the hydrometric station (Fig. 1).

The comparison of results of the research conducted in the years 2012-2013 with the multiannual 1986-2009 (Table 1) applied data covering 11 measurement seasons: 1986, 1987, 1988, 1989, 1990, 1993, 2001, 2002, 2005, 2006, and 2009. The analysed seasons flow rate was measured by current meter (Hega 2 and similar), while water levels respectively: 1986-2002 by float gauge; 2005-2009 by pressure water level logger. Due to the lack of multiannual continuity, lack of synchronicity, and varied duration of the measurement sequences in particular years (Table 1), as well as lack of some of hydrometric data resulting from a differing thematic profile of certain publications, the typical analysis of discharge variability based on mean monthly and annual values in particular years was impossible. Therefore, the analysis of the problem focused on daily discharge variability in particular research seasons, and differences between them. The comparison of analogical values was possible (not in all cases) only for the common summer period (from 13 July to 8 August) constituting only a part of the active hydrological period. Locating the obtained average values on the background of the active hydrological season provides a distorted image of discharge rate, specific runoff volume, and difference in the course of the phenomenon between the seasons. This should be considered in the case of comparisons of available data from particular years.

The description of discharge variability applied: the irregularity coefficient (expressed as the quotient of the maximum and minimum discharge in a given period), relative amplitude (ratio of the difference of extreme discharge values to the mean value), and variability coefficient (ratio of the standard deviation to the mean value) calculated for available measurement seasons and for the period from 13 July to 18 August a common for all seasons.

\section{The Scott River hydrology in the period 1986-2009}

Research by conducted in the Scott River catchment in the years 1986-2009 (Bartoszewski 1998, 2007, Bartoszewski et al. 2009, Kociuba et al. 2014) shows the mean multiannual discharge in the river of $0.98 \mathrm{~m}^{3} \cdot \mathrm{s}^{-1}$. The highest mean discharge was recorded in 2009 at a level of 2.00 $\mathrm{m}^{3} \cdot \mathrm{s}^{-1}$ (Table 1). The average daily discharge maximum was recorded on 7 August 1993, and amounted to $5.84 \mathrm{~m}^{3} \mathrm{~s}^{-1}$ at the mean value from the respective season of $1.78 \mathrm{~m}^{3} \cdot \mathrm{s}^{-1}$. The described season was also a period with the shortest observation series of 42 days. The lowest mean discharges, below $1.00 \mathrm{~m}^{3} \cdot \mathrm{s}^{-1}$, were determined in 1987 and 1988. In the same season (1988), on 7 October, the minimum mean daily discharge for the entire multiannual was recorded, amounting to $0.002 \mathrm{~m}^{3} \cdot \mathrm{s}^{-1}$. The maximum daily discharge in the period reached a value of $3.52 \mathrm{~m}^{3} \cdot \mathrm{s}^{-1}$. It was the longest measurement season lasting 100 days. In the years 1986-2009, the mean discharge in the Scott River increases both in the case of shorter, common measurement series (from 13 July to 8 August), and for full observation periods with varied duration (Table 1, Fig. 7).

The mean multiannual specific runoff in the Scott River amounts $97.6 \mathrm{dm}^{3} \cdot \mathrm{s}^{-1} \mathrm{~km}^{2}$ in the period 1986-2009 (966 mm; Bartoszewski 1998, 2007, Bartoszewski et al. 2009, Kociuba et al. 2014). The highest mean specific runoff summer season $\left(197.5 \mathrm{dm}^{3} \cdot \mathrm{s}^{-1} \mathrm{~km}^{2}\right)$ was recorded in 2009 , and the lowest $\left(82.8 \mathrm{dm}^{3} \cdot \mathrm{s}^{-1} \cdot \mathrm{km}^{2}\right)$ in 1988 . Total runoff covering 9 measurement seasons varied from 4.59 (1986) to $9.96 \mathrm{M} \mathrm{m}^{3}$ (2009), and the runoff 


\begin{tabular}{|c|c|c|c|c|c|c|c|c|c|c|c|c|c|c|c|}
\hline & 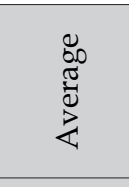 & तु & 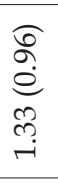 & 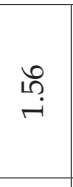 & $\begin{array}{l}\text { ర్ } \\
\text { ले }\end{array}$ & Iீ. & 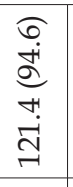 & 䒿 & 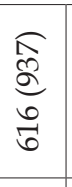 & $\stackrel{\leftrightarrow}{\stackrel{\leftrightarrow}{r}}$ & 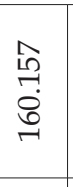 & $\begin{array}{l}\infty \\
\stackrel{\infty}{n} \\
\text { i }\end{array}$ & 今+ & $\begin{array}{l}0 \\
m \\
0\end{array}$ & 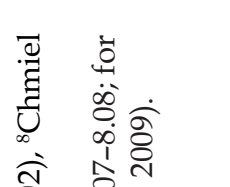 \\
\hline & 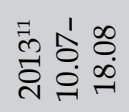 & શิ & $\stackrel{\text { }}{\stackrel{-}{r}}$ & $\stackrel{+H}{\stackrel{H}{r}}$ & $\begin{array}{l}3 \\
+\end{array}$ & $\stackrel{+}{\stackrel{+}{0}}$ & $\stackrel{\circ}{\stackrel{+}{\sigma}}$ & $\begin{array}{l}\overrightarrow{7} \\
\vec{\sigma}\end{array}$ & స్ & $\hat{\vartheta}$ & 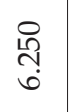 & શે & F! & 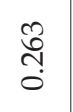 & 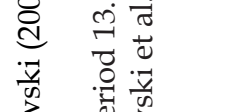 \\
\hline & 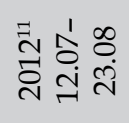 & $\stackrel{m}{m}$ & $\stackrel{8}{\circ}$ & $\stackrel{\sigma}{\sigma}$ & $\stackrel{\vec{\lambda}}{\vec{i}}$ & సે & $\begin{array}{l}0 \\
\infty \\
\infty\end{array}$ & $\begin{array}{l}0 \\
\dot{\infty}\end{array}$ & సે & $\stackrel{+}{\wedge}$ & 常 & $\underset{\stackrel{\infty}{i}}{\stackrel{i}{i}}$ & $\stackrel{\text { Fे }}{\circ}$ & लै & $\begin{array}{ll}0 & 0 \\
0 \\
0\end{array}$ \\
\hline & 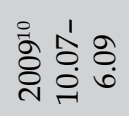 & ๙̊. & $\underset{\text { i }}{\stackrel{\text { i }}{ }}$ & $\stackrel{\vec{\lambda}}{\vec{\lambda}}$ & $\stackrel{\text { PH }}{\text { i }}$ & $\stackrel{\text { ळે }}{\rightarrow}$ & $\begin{array}{l}\stackrel{10}{\circ} \\
\text { مे }\end{array}$ & $\begin{array}{l}\text { ñ. } \\
\stackrel{0}{0}\end{array}$ & ळ & $\stackrel{\circ}{\circ}$ & 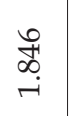 & $\begin{array}{l}\text { 员 } \\
\text { ڤִ } \\
0\end{array}$ & ֻ̊? & $\begin{array}{l}\stackrel{8}{8} \\
\stackrel{0}{0}\end{array}$ & 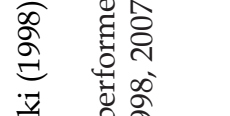 \\
\hline & 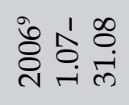 & भु & 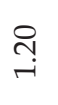 & $\stackrel{\text { ڤે }}{\rightarrow}$ & $\stackrel{\stackrel{2}{\vec{i}}}{\vec{i}}$ & 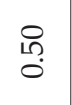 & $\begin{array}{l}\stackrel{0}{\infty} \\
\stackrel{\infty}{=}\end{array}$ & $\stackrel{\vec{m}}{\vec{m}}$ & గి & $\stackrel{3}{\stackrel{3}{0}}$ & $\begin{array}{l}8 \\
\stackrel{\infty}{+} \\
+\end{array}$ & 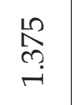 & $\begin{array}{l}\stackrel{2}{1} \\
\text { İ }\end{array}$ & 离 & 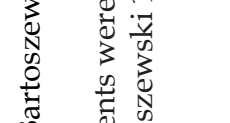 \\
\hline 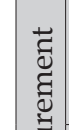 & 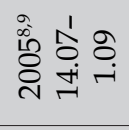 & 구 & 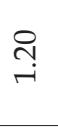 & $\begin{array}{l}\infty \\
\infty \\
0 \\
0\end{array}$ & $\begin{array}{l}\mathscr{Q} \\
\infty \\
\infty\end{array}$ & ڤึ? & $\begin{array}{l}\stackrel{\vec{े}}{\Rightarrow} \\
\vec{\Rightarrow}\end{array}$ & $\underset{\infty}{\infty}$ & : & $\vec{a}$ & 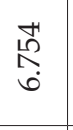 & $\begin{array}{l}\overrightarrow{\mathrm{d}} \\
\stackrel{\mathrm{N}}{ }\end{array}$ & $\begin{array}{l}\text { N̂̃ } \\
\text { O̦ }\end{array}$ & 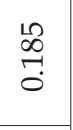 & 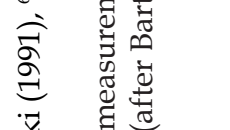 \\
\hline $\begin{array}{l}\tilde{\Xi} \\
\tilde{\Xi} \\
\tilde{q} \\
\overrightarrow{0}\end{array}$ & 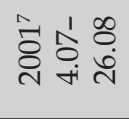 & $\begin{array}{l}8 \\
\qquad{ }^{\circ}\end{array}$ & $\underset{\sim}{F}$ & تُ & $\begin{array}{l}\infty \\
\cdots \\
\infty\end{array}$ & 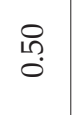 & مे & & $\stackrel{8}{8}$ & $\stackrel{m}{\sigma}$ & 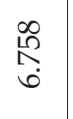 & $\begin{array}{l}\text { मn } \\
\text { in } \\
\text { in }\end{array}$ & ت્ટ் & $\begin{array}{l}\vec{\infty} \\
\stackrel{0}{0} \\
0\end{array}$ & 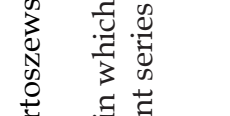 \\
\hline $\overrightarrow{\tilde{\Omega}}$ & 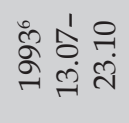 & تُ & $\stackrel{\infty}{\stackrel{\infty}{\sim}}$ & $\stackrel{\Omega}{\leftrightarrow}$ & 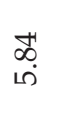 & $\stackrel{l}{\sharp}$ & 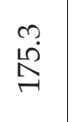 & 명. & 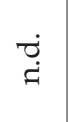 & ت્ટ் & $\begin{array}{l}\text { Lి } \\
\stackrel{0}{0} \\
\text { î }\end{array}$ & $\begin{array}{l}\widehat{\widehat{o}} \\
\text { ले }\end{array}$ & $\begin{array}{l}\stackrel{L}{n} \\
\stackrel{n}{n} \\
0\end{array}$ & 草 & 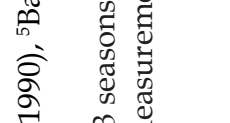 \\
\hline & 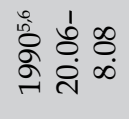 & $\begin{array}{l}\infty \\
\infty \\
10\end{array}$ & $\stackrel{\text { ले }}{\rightarrow}$ & $\underset{\sim}{\mathbb{H}}$ & 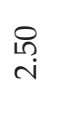 & $\stackrel{8}{\circ}$ & $\stackrel{\infty}{\stackrel{\infty}{\oplus}}$ & $\begin{array}{l}\vec{H} \\
\vec{H}\end{array}$ & 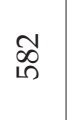 & $\begin{array}{l}\underset{-}{ت} \\
\vec{\nabla}\end{array}$ & $\begin{array}{l}\infty \\
\infty \\
\infty \\
\infty\end{array}$ & م̂ & $\begin{array}{l}\hat{\infty} \\
\text { ஸ̣ }\end{array}$ & तु & 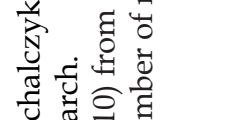 \\
\hline & 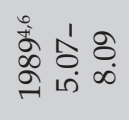 & $\vec{\infty}$ & $\stackrel{\text { ڤొ }}{\longrightarrow}$ & $\underset{\mathrm{i}}{\stackrel{F}{H}}$ & 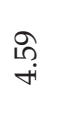 & $\stackrel{\hat{O}}{0}$ & $\begin{array}{l}0 \\
\stackrel{0}{10} \\
\stackrel{0}{\sim}\end{array}$ & $\begin{array}{l}\text { Na } \\
\infty \\
\text { ָे }\end{array}$ & $\begin{array}{l}\infty \\
\infty \\
\infty\end{array}$ & $\begin{array}{l}\stackrel{m}{\oplus} \\
\stackrel{\oplus}{\sigma}\end{array}$ & 总 & $\begin{array}{l}\bar{\delta} \\
\text { ì }\end{array}$ & đ্̉ & $\begin{array}{l}\text { के } \\
\text { đִ } \\
0\end{array}$ & 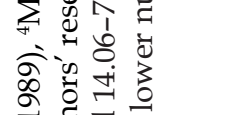 \\
\hline & 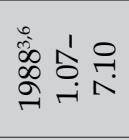 & $\stackrel{\overbrace{}}{\curvearrowright}$ & $\stackrel{2}{\circ}$ & $\stackrel{\text { மே }}{\rightarrow}$ & $\begin{array}{l}\text { N̂ } \\
\text { ल) }\end{array}$ & §ิ & $\begin{array}{l}\infty \\
\text { న } \\
\infty\end{array}$ & 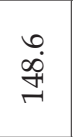 & $\stackrel{\infty}{R}$ & $\stackrel{\Upsilon}{\wedge}$ & $\begin{array}{l}8 \\
8 \\
0 \\
10 \\
\frac{1}{7}\end{array}$ & $\begin{array}{l}\infty \\
\stackrel{\infty}{0} \\
\stackrel{\infty}{\infty}\end{array}$ & $\begin{array}{l}\text { 蒙 } \\
\text { O }\end{array}$ & तु్ & 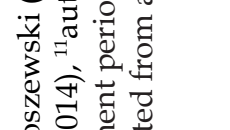 \\
\hline & 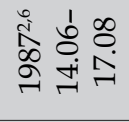 & $\begin{array}{l}\text { ले } \\
\text { மे }\end{array}$ & ః̊ & مै & 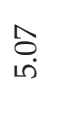 & 궁 & ث̊t & 弪 & กิ & $\underset{\infty}{N}$ & $\begin{array}{l}\frac{\infty}{m} \\
\stackrel{N}{2}\end{array}$ & 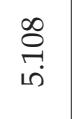 & 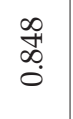 & 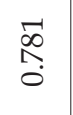 & 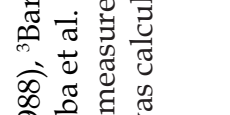 \\
\hline & 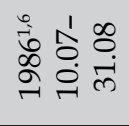 & ڤ్ & 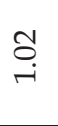 & 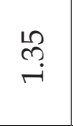 & $\begin{array}{l}\text { के } \\
\text { ले }\end{array}$ & $\begin{array}{l}\stackrel{2}{0} \\
\text { İ }\end{array}$ & ڤ્ণ & $\begin{array}{l}\stackrel{0}{\infty} \\
\stackrel{\leftrightarrow}{-}\end{array}$ & 䆘 & $\stackrel{L}{\infty}$ & $\begin{array}{l}\text { लू' } \\
\text { ì }\end{array}$ & $\underset{8}{0}$ & 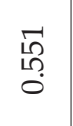 & $\begin{array}{l}\text { के } \\
\text { ఝై } \\
0\end{array}$ & 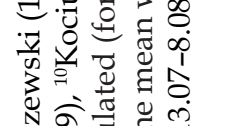 \\
\hline & 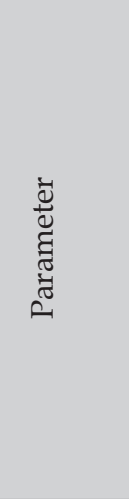 & 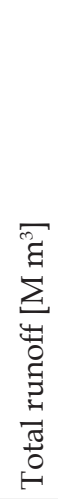 & 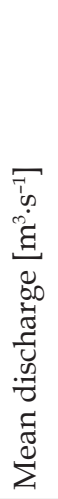 & 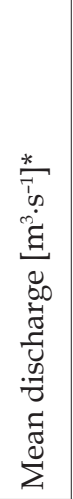 & 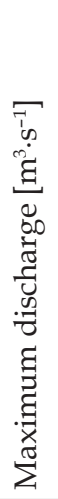 & 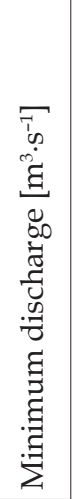 & 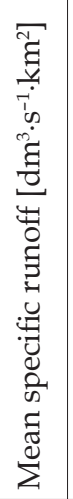 & 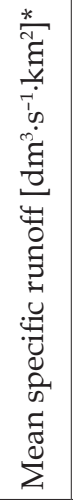 & 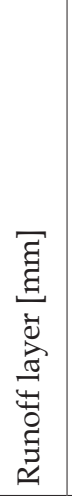 & 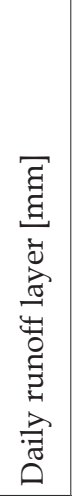 & 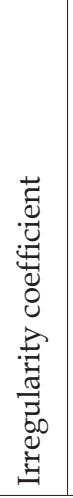 & 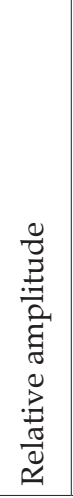 & 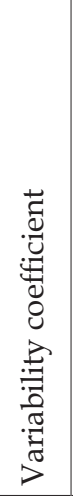 & 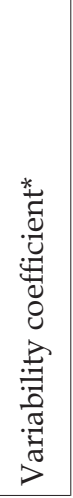 & 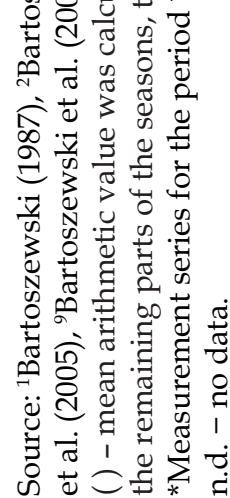 \\
\hline
\end{tabular}


layer amounted to 452 and $984 \mathrm{~mm}$, respectively (Table 1).

Characteristics of discharge variability in the period 1986-2009 show evident variability. Values of the discharge irregularity coefficient calculated for each measurement seasons (with varied duration) in particular years show high variability, and range from 1.846 (2009) to 1759.000 (1988). The relative amplitude reached the minimum value of 0.550 in 2009 , and a maximum of 5.108 in 1987. The variability coefficient was in the range from 0.245 (2009) to 0.848 (1987). For the common measurement period, (lasting from 13 July to 8 August), the index reached the minimum value (0.181) in 2001, and the highest (0.781) in 1987 (Table 1).

\section{Results of investigations in 2012 and 2013}

The mean daily air temperature amounted 4.6 and $5.9^{\circ} \mathrm{C}$ in the summers 2012 and 2013 respectively. The highest mean daily temperature in 2012 was recorded on 22 July, and it amounted $7.1^{\circ} \mathrm{C}$, and the lowest one - on 21 August, amounting $2.0^{\circ} \mathrm{C}$. In 2013 , the highest mean daily air temperature amounted $8.6^{\circ} \mathrm{C}$ (17 August), and the lowest $2.7^{\circ} \mathrm{C}$ (13 August) (Fig. 3 and 4).

In terms of atmospheric precipitation, research seasons 2012 and 2013 differed from each other. Total atmospheric precipitation in the summer 2012 amounted $26.7 \mathrm{~mm}$ and in the summer 2013

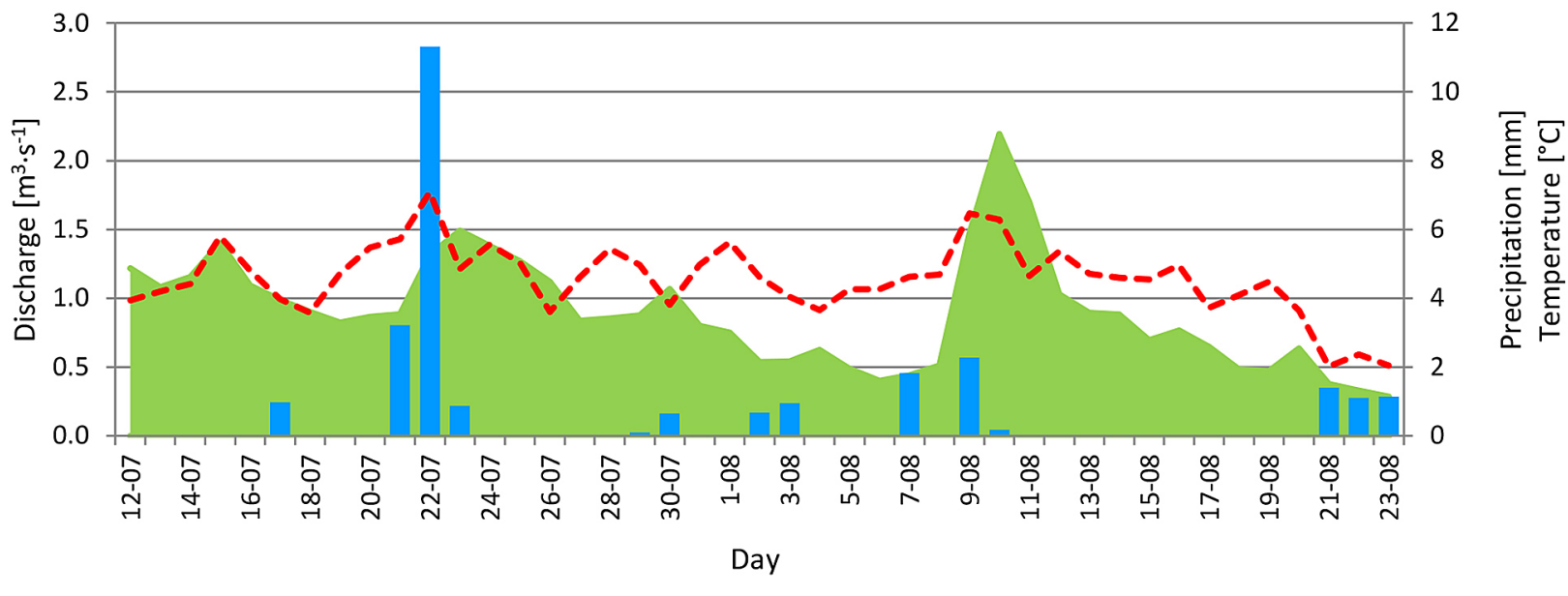

Discharge Precipitation ----Temperature

Fig. 3. Course of mean daily discharges in the Scott River in comparison to daily precipitation and air temperature in the measurement period 2012.

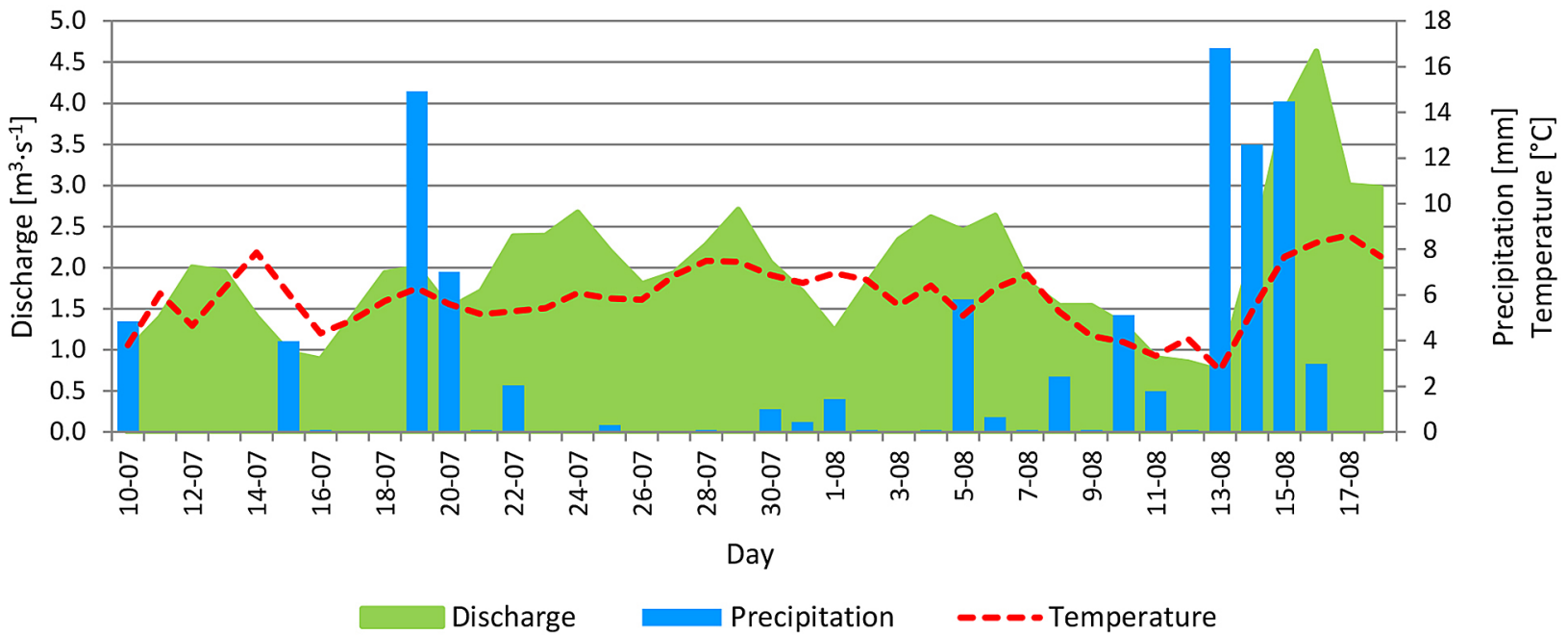

Fig. 4. Course of mean daily discharges in the Scott River in comparison to daily precipitation and air temperature in the measurement period 2013. 

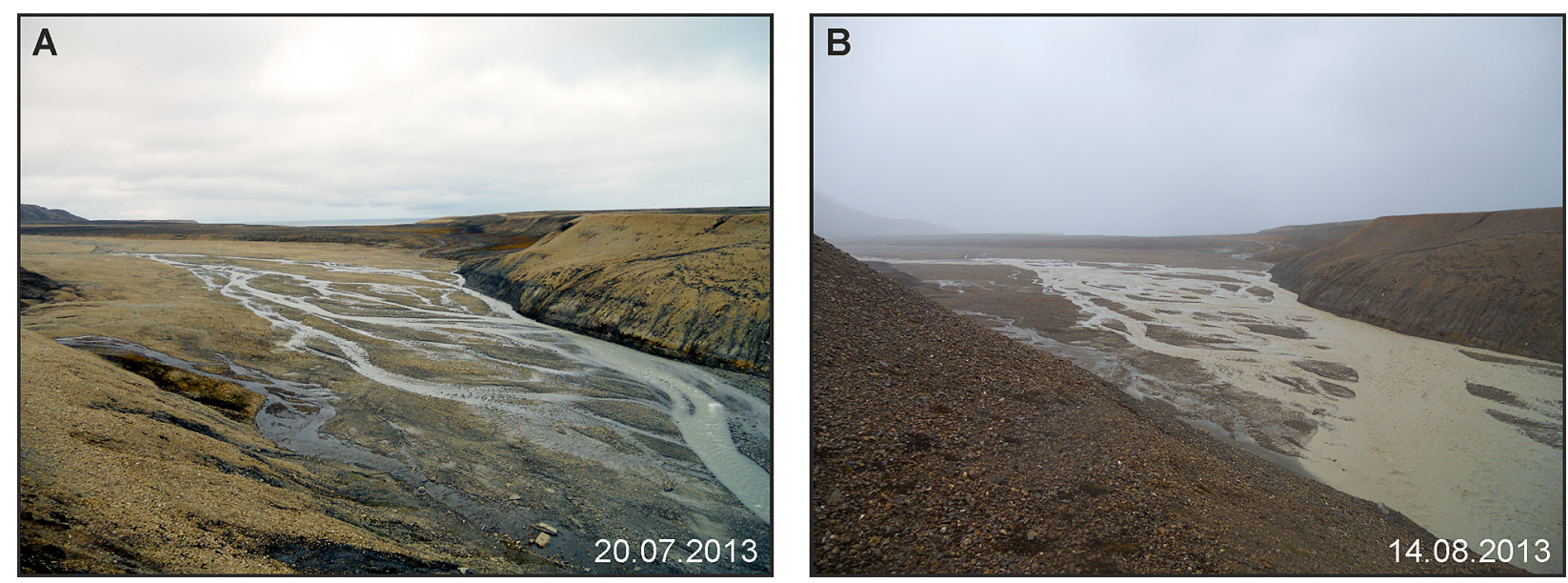

Fig. 5. Flooding in the gorge section of the Scott River in the research season 2013: A - before the flooding, $\mathrm{B}$ - during the flooding.
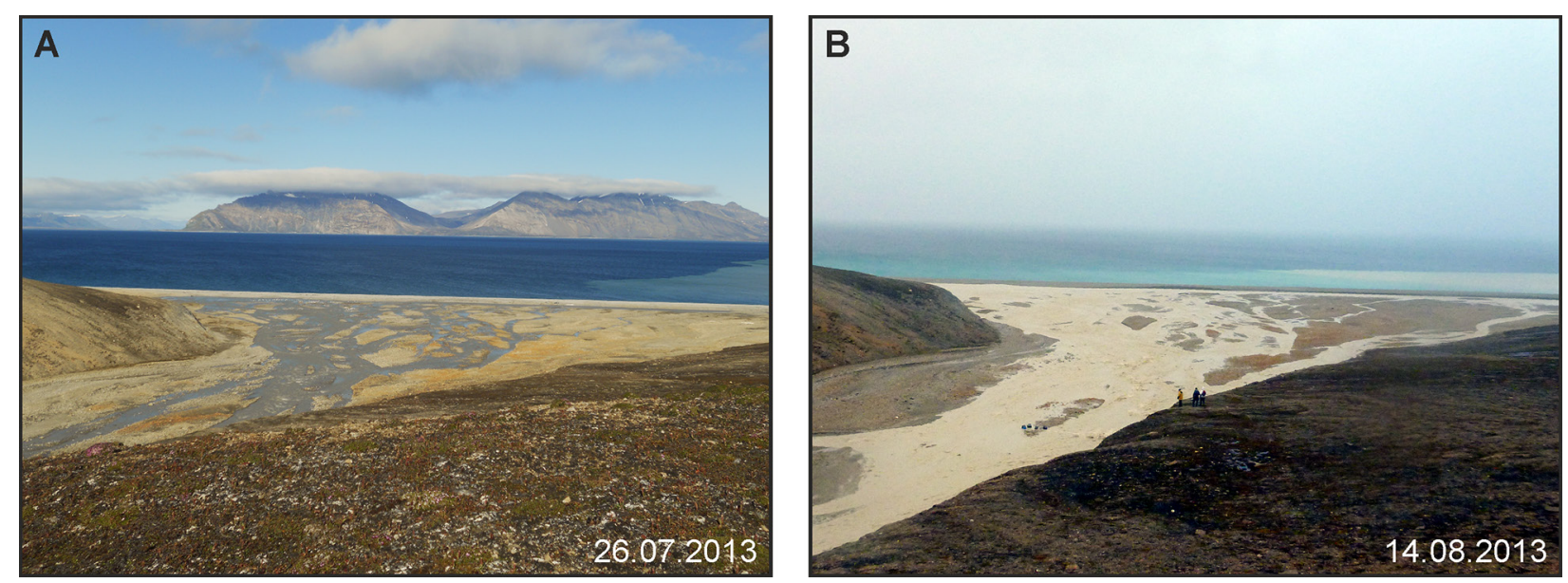

Fig. 6. Flooding in the mouth section of the Scott River in the research season 2013: A - before the flooding, $\mathrm{B}$ - during the flooding.

reached $98.9 \mathrm{~mm}$. In season 2012, atmospheric precipitation occurred seldom, and were distinguished by high variability. Twelve days with precipitation were recorded, including six days with precipitation above $1.0 \mathrm{~mm}$. The highest precipitation total was recorded on 22 July. It amounted to $11.3 \mathrm{~mm}$, corresponding to almost half of the precipitation total recorded in season 2012. In season 2013, precipitation was more abundant, and occurred more frequently than in the preceding year. 26 days with precipitation were recorded, including 15 days with precipitation above $1.0 \mathrm{~mm}$. The highest precipitation occurred on 13 August, and amounted to $16.8 \mathrm{~mm}$. In the period from 13 to 16 August, it constituted almost $50 \%(46.9 \mathrm{~mm})$ of the precipitation total for 2013 (Fig. 3 and 4).

The course of hydrological conditions in research seasons 2012 and 2013 was variable. The mean river discharge amounted to 0.90 and 1.96 $\mathrm{m}^{3} \mathrm{~s}^{-1}$, respectively, which is equivalent to specific runoff of 88.6 and $194.0 \mathrm{dm}^{3} \cdot \mathrm{s}^{-1} \mathrm{~km}^{2}$ (Table 1).

The analysis of daily discharges in the summer season 2012 shows that from the beginning of the measurement period, a gradual decrease in discharges was recorded on 6 August, reaching the value of $0.40 \mathrm{~m}^{3} \cdot \mathrm{s}^{-1}$. On consecutive days, an increase was observed with discharge culmination on 10 August amounting to $2.19 \mathrm{~m}^{3} \cdot \mathrm{s}^{-1}$. The lowest discharges of $0.29 \mathrm{~m}^{3} \mathrm{~s}^{-1}$ were recorded at the end of the research period on 23 August (Table 1, Fig. 3). In the next research season (2013), the discharge curve shows the opposite situation. A constant increase in discharges is observed with several secondary floodings and maximum flooding on 16 August $\left(4.63 \mathrm{~m}^{3} \cdot \mathrm{s}^{-1}\right.$; Fig. 5 and 6), preceded by a period of the lowest water level recorded on 13 August, distinguished 


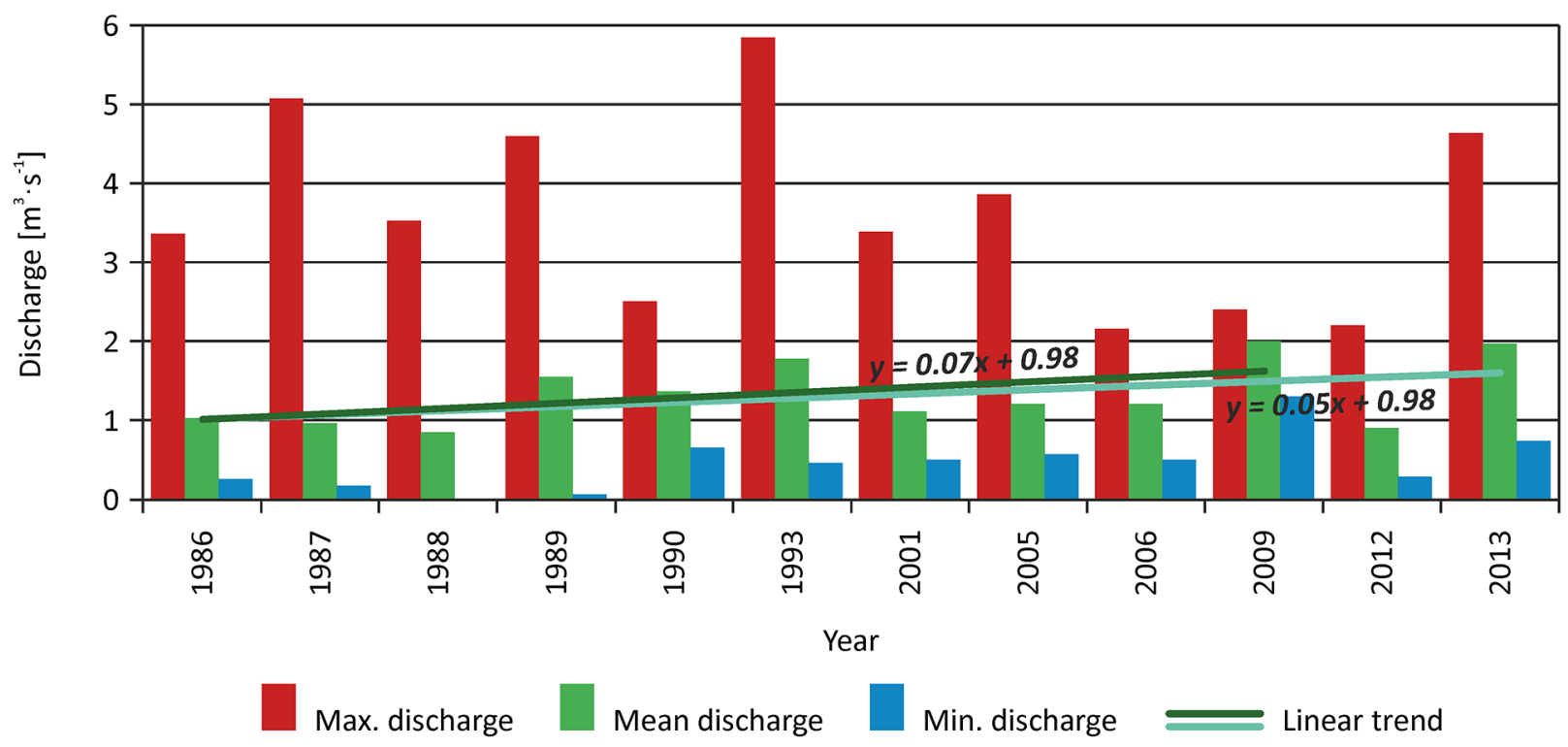

Fig. 7. Mean seasonal discharges in the Scott River in comparison to maximum and minimum discharges in the years 1986-2013.

by a decrease in discharge to the value of 0.74 $\mathrm{m}^{3} \cdot \mathrm{s}^{-1}$ (Table 1, Fig 4).

Total runoff in 2012 and 2013 amounted to 3.33 and $6.79 \mathrm{M} \mathrm{m}^{3}$, respectively, and the runoff layer to 329 and $671 \mathrm{~mm}$. For the longest common measurement period corresponding with the multiannual 1986-2009, lasting from 13 July to 8 August, the value amounted to 2.12 and 4.51 $\mathrm{M} \mathrm{m}^{3}$, and 209 and $446 \mathrm{~mm}$, respectively.

Discharge variability calculated for measurement periods expressed as variability coefficient amounted to 0.449 in 2012, and 0.411 in 2013. The value for the same observation period was 0.343 in 2012 and 0.263 in the following year (Table 1).

The analysis of the collected material shows that the thermal conditions have the strongest impact on runoff values which is expressed in high values of the correlation coefficient between mean daily discharge and mean daily air temperature. It amounts 0.60 in 2012 and 0.67 in 2013. Rainfall inconsiderably modify runoff volume however there occured several floods caused by rain. The influence of rainfall on the river runoff was observed in periods with higher total values (2012 - $15.4 \mathrm{~mm}$; 2013 - $46.9 \mathrm{~mm}$ ).

Considering data from all of the research seasons (1986-2013), multiannual mean daily discharge turned out to be somewhat lower than for multiannual 1986-2009. It amounted to 0.96 $\mathrm{m}^{3} \cdot \mathrm{s}^{-1}(937 \mathrm{~mm})$. Also multiannual mean daily discharge for the same measurement period (from 13 July to 8 August; covering 11 seasons) was lower, amounting to $1.56 \mathrm{~m}^{3} \cdot \mathrm{s}^{-1}$ (Table 1). The analysis of all of the measurement seasons showed an increasing tendency of mean annual discharges of the studied river somewhat higher than in the multiannual 1986-2009 (Fig. 7).

\section{Discussion}

Results of studies on glacial hydrology provide information concerning the consequence of global climate change and its hydrological responses (e.g. Peterson et al. 2002, Syvitski 2002, Gordeev 2006, Hasholt et al. 2006, Holland et al. 2007). During the 21st century, Lewis and Lamoureux (2010) predicted increases in runoff in a small catchment in the Canadian Arctic Archipelago, resulting in greater discharge variability. The general growth trend was confirmed by the results of a current study in the proglacial Scott River.

There were considerable differences in the river runoff in the Scott River catchment in the summers 2012 and 2013. The mean discharge in season 2012 was two times lower $\left(0.91 \mathrm{~m}^{3} \cdot \mathrm{s}^{-1}\right)$ than in 2013 and it constitutes more than half of the mean river runoff in the years $1986-2009\left(1.56 \mathrm{~m}^{3} \cdot \mathrm{s}^{-1}\right)$ for the same measurement period from 13 July to 8 August. The mean multiannual discharge for the entire active hydrological season was 
at a level of $0.98 \mathrm{~m}^{3} \cdot \mathrm{s}^{-1}(966 \mathrm{~mm}$; Bartoszewski 1998, 2007, Bartoszewski et al. 2009, Kociuba et al. 2014). Considering discharges from the years 2012-2013, the mean multiannual value changes inconsiderably, amounting to $0.96 \mathrm{~m}^{3} \cdot \mathrm{s}^{-1}$, i.e. 937 $\mathrm{mm}$.

Mean multiannual runoff in glacial catchments of Spitsbergen is very variable, and amounts from 500 to $1800 \mathrm{~mm}$ (Bartoszewski 1998, 2007, Killingtveita et al. 2003, Sund et al. 2008, Hodgkins et al. 2009, Sobota 2013, Majchrowska et al. 2015). Factors determining such high variability is the contribution of area occupied by the glacier, and course of meteorological conditions affecting the ablation rate. Values of multiannual runoff obtained by the authors are within the above range, Comparable runoff (in the years 1990-2001), amounting to $1091 \mathrm{~mm}$, was obtained by Killingtveita et al. (2003) in the Bayelva catchment $\left(31 \mathrm{~km}^{2}\right)$, occupied by the glacier in $55 \%$. Similar runoff conditions - $1073 \mathrm{~mm}$ for 1999, were observed by Hodgkins et al. (2009) in the Finsterwalderbreen catchment $\left(66 \mathrm{~km}^{2}\right)$ glaciated in $66 \%$. Considerbaly lower values of mean multiannual runoff (1997-2010) are provided by Sobota (2013) determining runoff of $540 \mathrm{~mm}$ in the Waldemar River catchment with an area of almost $5 \mathrm{~km}^{2}$ and contribution of glaciated area of 50\%. The same runoff (in the years 1990-2001) amounting to $540 \mathrm{~mm}$ is also mentioned by Killingtveita et al. (2003) for De Geerelva $(79$ km² $10 \%)$ and Endalen $\left(29 \mathrm{~km}^{2} ; 20 \%\right)$. Low runoff values at a level of approximately $300 \mathrm{~mm}$ for a part of the summer sezon 1985 in the Ebbaelva catchment wirth an area of $51 \mathrm{~km}^{2}$ and glaciation of $52 \%$ are provided by Kostrzewski et al. (1989). The Werenskiold glacier catchment with an area of $44 \mathrm{~km}^{2}$ and glaciation of $62 \%$ in the years 2007-2012 showed runoff at a level of $1800 \mathrm{~mm}$ (Majchrowska et al. 2015). Also in the earlier period (1980), runoff showed high values - $1876 \mathrm{~mm}$ (Leszkiewicz 1987).

The analysis of all measurement seasons (period from 1986 to 2013) showed that the highest discharges (approximately $1.50 \mathrm{~m}^{3} \cdot \mathrm{s}^{-1}$ ) occurred in the second and third decade of July, and the first and second decade of August. A growing tendency for mean annual discharges predicted for Arctic Archipelago by Lewis and Lamoureux (2010) and determined in the scope of a field study (Killingtveita et al. 2003, Majchrowska et al. 2015) was also observed in the analysed river, where mean discharge increase amounted to approximately $60 \%$ over a 27 -year period (Fig. 7 ).

The analysed summer seasons 2012 and 2013 were distinguished by varied runoff dynamics. In 2012, low water stages were maintained over the majority of the time, separated by three flood seasons (maximum flooding amounted to $\left.2.19 \mathrm{~m}^{3} \mathrm{~s}^{-1}\right)$. In the following year, high water levels were maintained for a longer period, with an extreme flooding at the end of the measurement season $\left(4.63 \mathrm{~m}^{3} \cdot \mathrm{s}^{-1}\right)$, whereas periods of low water level were rare. Similar variability is observed in the discussed multiannual 1986-2009, involving the occurrence of less and more wet seasons with periods with particularly high water level increases and values approximate to those recorded in 2013. In the analysed period, these were years: 1987, 1989, 1993, and 2005 (Bartoszewski 1988, 1998, Michalczyk 1990, Bartoszewski et al. 2009).

Considerable variability of discharge rate in the Scott River, both in the case of the majority of particular measurement seasons and multiannual course one can observe. The discharge variability coefficient for the summer season lasting from 13 July to 8 August in the summers 2012 and 2013 amounted 0.343 and 0.263 , respectively, and in the analogical period for the years 1986-2001, it ranged from 0.180 to 0.780 (Bartoszewski 1989, $1991,1998,2002)$. The lowest values of the variability coefficient in the analysed period suggest relatively even river discharge. They occurred in seasons (period from 13 July to 8 August) in which no considerable foods or extreme low-water periods were recorded.

Research seasons 2012 and 2013 showed different meteorological conditions. Year 2012 was much cooler and drier than the following year, as reflected in the volume of water discharged from the catchment, more than twice higher in the wet season (2013; runoff layer $446 \mathrm{~mm}$ ) than in the dry season (runoff layer $209 \mathrm{~mm}$ ) in the comparable period. In the period 1986-2013, mean annual runoff layer for seasons with different measurement duration varied from 452 to $984 \mathrm{~mm}$, and the multiannual mean value calculated for the longest active hydrological period (Bartoszewski 1998, 2007, Bartoszewski et al. 2009, Kociuba et al. 2014) amounted to $937 \mathrm{~mm}$ (Table 1). 
Runoff in the Scott River is particularly determined by meteorological conditions affecting the intensity of ablation of the glacier. The most significant role is played by: air temperature, distribution and intensity of atmospheric precipitation, and the foehn effect, or several phenomena combined (Bartoszewski 1988, 1998). In the period analysed based on the literature (1986-2009) and in research seasons 2012 and 2013, a correlation was determined between the course of daily air temperature and river runoff, resulting in intensive glacial ablation (correlation coefficient was 0.60 in 2012 and 0.67 in 2013). Moreover, in 2013, an extremely high flood was recorded due to simultaneous occurrence of air temperature increase and intensive rainfall over a short period of time, as confirmed also studies by other authors (Piasecki and Pulina 1975, Bartoszewski 1988, 1998, Szczepanik and Marciniak 1989, Rachlewicz 2007, Sobota and Nowak 2012, Sobota 2013).

\section{Conclusions}

The studied proglacial Scott River catchment with almost a 30-year observation sequence constitutes one of the most thoroughly hydrologically investigated small glacial catchments of western Spitsbergen. The presented results of measurements from summer seasons 2012-2013 permitted a comparison with the 11-year observation period, and analysis of changes occurring during the culmination of the melt season (from July 12 to August 23).

Research conducted in melt seasons 2012-2013 as well as study results from the years 1986-2009 confirm the constant increasing trend anticipated for small glacial catchments of Arctic archipelagos by Lewis and Lamoureux (2010). A characteristic feature of such changes are greater discharge fluctuations - daily, seasonal, as well as multiannual.

The recorded seasonal irregularity of hydrological conditions resulted from high variability of meteorological conditions in the analysed period. Melt season 2012 was cooler and dry, and 2013 warmer and more humid than average.

The primary factor determining runoff is air temperature. The research confirmed the positive correlation of mean daily air temperature and discharge (0.60 in 2012 and 0.67 in 2013). The amount of precipitation is of lower importance, although the highest discharges (including flood discharges) are usually initiated as a result of combination of both of the factors at the same time.

\section{Acknowledgements}

The study was conducted in the scope of the $24^{\text {th }}$ and $25^{\text {th }}$ Polar Expeditions of the Marie Curie-Skłodowska University in Lublin to Spitsbergen, implementing grant of the National Science Centre Mechanisms of fluvial transport and delivery of sediment to the Arctic river channels with different hydrological regime (SW Spitsbergen) No. 2011/01/B/ST10/06996.

\section{References}

Baranowski S., 1977. Subpolarne lodowce Spitsbergenu na tle klimatu tego region (The subpolar glaciers of Spitsbergen seen against the climate of this region). Acta Universitatis Wratislaviensis 393: 1-157.

Baranowski S., Głowicki B., 1975. Meteorological and hydrological investigations in the Homsund region made in 1970. Acta Universitatis Wratislaviensis 251: 54-59.

Bartoszewski S., 1987. Dynamika odpływu powierzchn-iowego w zlewniach rzek lodowcowych Scotta, Blomli i Tjörn podczas lata 1986 (Spitsbergen Zachodni) (Surface runoff dynamics of the basins Scott, Blomli and Tjörn glacier rivers in the summer 1986 (West Spitsbergen)). In: Repelewska-Pękalowa J., Harasimiuk M., Pękala K. (eds), XIV Sympozjum Polarne. Wydawnictwo UMCS, Lublin: 151-156.

Bartoszewski S., 1988. Warunki kształtowania się odpływu w zlewni Scotta (Zachodni Spitsbergen) (The outflow conditions in the basin of the Scott's River (West Spitsbergen)). In: Repelewska-Pękalowa J., Harasimiuk M., Pękala K. (eds), Wyprawy Geograficzne UMCS na Spitsbergen 1986-1988. Wydawnictwo UMCS, Lublin: 115-122.

Bartoszewski S., 1989. Charakterystyka odpływu ze zlewni lodowca Scotta (Zachodni Spitsbergen) w 1988 r. (Characterization of outflow from the Scott Glacier basin, West Spitsbergen). In: Repelewska-Pękalowa J., Pękala K. (eds), Wyprawy Geograficzne UMCS na Spitsbergen 19881989. Wydawnictwo UMCS, Lublin: 61-67.

Bartoszewski S., 1991. Ablation of Scott Glacier and its role in river flow formation. In: Repelewska-Pękalowa J., Pękala K. (eds), Wyprawy geograficzne UMCS na Spitsbergen 1991. Wydawnictwo UMCS, Lublin: 107-121.

Bartoszewski S., 1998. Reżim odptywu rzek Ziemi Wedel Jarlsberga (Spitsbergen) (Flow regime of rivers in Wedel Jarlsberg Land (Spitsbergen)). Rozprawy Habilitacyjne, LX. Wydawnictwo UMCS, Lublin.

Bartoszewski S., 2002. Odpływ ze zlewni Lodowca Scotta (Spitsbergen) w sezonie letnim 2001 r. (Runoff from the Scott Glacier catchment (Spitsbergen) in the summer season of 2001). In: Kostrzewski A., Rachlewicz G. (eds), 
Polish Polar Studies. Bogucki Wydawnictwo Naukowe, Poznań: 65-72.

Bartoszewski S., 2007. Reżim odpływu rzeki Scotta (Spitsbergen) (Runoff regime of the Scott River (Spitsbergen)). In: Michalczyk Z. (ed.), Obieg wody w środowisku naturalnym i przeksztatconym. Badania Hydrograficzne w Poznawaniu Środowiska, 8. Wydawnictwo UMCS, Lublin: 65-71.

Bartoszewski S., Gluza A., Siwek K., Zagórski P., 2009. Temperature and rainfall control of outflow from the Scott Glacier catchment (Svalbard) in the summer of 2005 and 2006. Norsk Geografisk Tidsskrift - Norwegian Journal of Geography 63(2): 107-114. DOI: 10.1080/00291950902907785.

Brykała D., Araźny A., 2002. Wpływ warunków meteorologicznych na kształtowanie się odpływu powierzchniowego na lodowcu Waldemara (NW Spitsbergen) latem 1997 roku (Effect of meteorological conditions on the surface outflow at the Waldemar Glacier, NW Spitsbergen, in the summer of 1997). Problemy Klimatologii Polarnej 10: 139-158.

Chmiel S., Bartoszewski S., Siwek K., Sposób J., 2012. Chemical and mechanical denudation rates in the Scott River catchment (Svalbard) during the summer season 2005. Annales UMCS, Geographia, Geologia, Mineralogia et Petrographia 67(1): 93-107. DOI: 10.2478/v10066-012-0006-7.

Collins D.N., 1979. Quantitative determination of the subglacial hydrology of two Alpine glaciers. Journal of Glaciology 23: 347-362.

Dallmann W.K., Hjelle A., Ohta Y., Salvigsen O., Bjornerud M.G., Hauser E.C., Maher H.D., Craddock C., 1990. Geological map of Svalbard 1:100 000, sheet B 11 G, Van Keulenfjorden. Norsk Polarinstitutt, Oslo.

Flood B., Nagy J., Winsnes T.S., 1971. Geological map of Svalbard 1:500 000, sheet 1 G, Spitsbergen southern part. Norsk Polarinstitutt, Oslo.

Głowicki B., 1982. Some hydrological phenomena observed in the outflow from the Werenskiold Glacier Basin. Spitsbergen Expeditions IV. Acta Universitatis Wratislaviensis 525: 49-56.

Gordeev V.V., 2006. Fluvial sediment flux to the Arctic Ocean. Geomorphology 80: 94-104. DOI: 10.1016/j.geomorph.2005.09.008.

Grześ M., Sobota I., 2000. Winter snow accumulation and discharge from the Waldemar Glacier, northwestern Spitsbergen in 1996-1998. Polish Polar Research 21(1): 19-32.

Hagen J.O., Etzelmuller B., Nuttall A.-M., 2000. Runoff and drainage pattern derived from digital elevation models, Finsterwalderbreen, Svalbard. Annals Glaciology 31: 147152. DOI: $10.3189 / 172756400781819879$.

Hasholt B., Bobrovitskaya N., Bogen J., McNamara J., Mernild S.H., Milburn D., Walling D.E., 2006. Sediment transport to the Arctic Ocean and adjoining cold oceans. Hydrology Research 37: 413-432. DOI: 10.2166/nh.2006.023.

Hodgkins R., 1997. Glacier hydrology in Svalbard, Norwegian high arctic. Quaternary Science Reviews 16(9): 957973. DOI: 10.1016/s0277-3791(97)00032-2.

Hodgkins R., Tranter M., Dowdeswell M.A., 2004. The characteristics and formation of a high-arctic proglacial icing. Geografiska Annaler Series A, Physical Geography 86(3): 265275. DOI: $10.1111 /$ j.0435-3676.2004.00230.x.

Hodgkins R., Cooper R., Wadham J., Tranter M., 2009. The hydrology of the proglacial zone of high - Arctic glacier (Finsterwalderbreen, Svalbard). Atmospheric and surface water fluxes. Journal of Hydrology 378(1): 150-160. DOI: 10.1016/j.jhydrol.2009.09.020.
Holland M.M., Finnis J., Barnett A.P., Serreze M.C., 2007. Projected changes in Arctic Ocean freshwater budgets. Journal of Geophysical Research 112, G04S55: 1-13. DOI: 10.1029/ 2006JG000354.

Killingtveit Å, Pettersson L.-E., Sand K., 2003. Water balance investigations in Svalbard. Polar Research 22(2): 161-174. DOI: $10.3402 /$ polar.v22i2.6453.

Klæboe H., 1948. Hydrological Conditions in Norway. Norwegian Journal of Geography 20(1): 21-31.

Kociuba W., Janicki G., 2014. Continuous measurements of bedload transport rates in a small glacial river catchment in the summer season (Spitsbergen). Geomorphology 212: 58-71. DOI: 10.1016/j.geomorph.2013.05.001.

Kociuba W., Janicki G., 2015a. Changeability of movable bed-surface particles in natural, gravel-bed channels and its relation to bedload grain size distribution (Scott River, Svalbard). Geografiska Annaler Series A, Physical Geography 97(3): 1-15. DOI: 10.1111/geoa.12090.

Kociuba W., Janicki G., 2015b. Spatiotemporal variability of the channel pattern of High Arctic proglacial rivers. In: Duncan N. (ed.), Fluvial Geomorphology and Riparian Vegetation: Environmental Importance, Functions and Effects on Climate Change. Nova Science Publishers, Inc., New York: 53-80.

Kociuba W., Janicki G., Siwek K., 2014. Variability of sediment transport in the Scott River catchment (Svalbard) during the hydrologically active season of 2009. Quaestiones Geographicae 33(1): 39-49. DOI: 10.2478/quageo-2014-0011.

Kociuba W., Krząstek P., Superson J., 2015. Combining GPSRTK and rephotographic methodologies for the assessment of transformations of the ephemeral landforms of the near foreland of a valley glacier (Scottbreen, Svalbard). Zeitschrift für Geomorphologie 60 (Suppl. 3): 029-044.

Krenke A.N., Khodakov V.G., 1966. On the relationship between the surface melting of glaciers with air temperature. Data of Glaciological Studies 12: 153-164.

Kostrzewski A., Kaniecki A., Kapuściński J., Klimaczak R., Stach A., Zwoliński Z., 1989. The dynamics and rate of denudation of glaciated and non-glaciated catchments, central Spitsbergen. Polish Polar Research 10: 317-367.

Kuziemski J., 1959. Niektóre wyniki obserwacji nad wodami lądowymi na Spitsbergenie w lecie 1958 roku (Some results of observations of inland waters on Spitsbergen in summer 1958). Przeglad Geofizyczny 4(3/4): 179-197.

Leszkiewicz J., 1982. Dynamika odpływu powierzchniowego zlewni potoku lodowca Nordfall (południowy Spitsbergen) (Surface run-off dynamics in the basin of the Nordfall Glacier stream, South Spitsbergen). Prace Naukowe Uniwersytetu Ślaskiego 543: 60-76.

Leszkiewicz J., 1987. Charakterystyczne cechy zlewni polarnych oraz próba modelowania statystycznego topnienia śniegu i odpływu ablacyjnego w zachodniej części Spitsbergenu (Characteristic features of the polar basins and an attempt of statistical modelling of the snow melting and ablation run-off in the western part of Spitsbergen). Prace Naukowe Uniwersytetu Ślaskiego 920. Wydawnictwo UŚ, Katowice.

Lewis T., Lamoureux S.F., 2010. Twenty-first century discharge and sediment yield predictions in a small high Arctic watershed. Global and Planetary Change 71(1): 2741. DOI: 10.1016/j.gloplacha.2009.12.00.

Majchrowska E., Ignatiuk D., Jania J., Marszałek H., Wąsik M., 2015. Seasonal and interannual variability in runoff from the Werenskioldbreen catchment, Spitsber- 
gen. Polish Polar Research 36(3): 97-224. DOI: 10.1515/ popore-2015-0014.

Marciniak K., Marszelewski W., 1990. Kształtowanie się odpływu w obrębie lodowca Elizy (NW Spitsbergen) w zależności od warunków pogodowych i ablacji $\mathrm{w}$ okresie lata polarnego (Formation of drainage within the Elise Glacier (NW Spitsbergen) in relations to Weather Conditions and ablation during the polar summer). Acta Universitatis Nicolai Copernici, Geografia 22(73): 125161.

Michalczyk Z., 1990. Hydrological characteristics of Calypsostranda. In: Repelewska-Pękalowa J., Pękala K. (eds), Wyprawy Geograficzne na Spitsbergen. UMCS, Lublin: 5-91.

Østrem G., 1973. Runoff forecasts for highly glacierized basins. The role of snow and ice in hydrology. International Association of Hydrological Sciences 107: 1111-1129.

Pardè M., 1957. Rzeki (Rivers). Wydawnictwo PWN, Warszawa.

Pereyma J., 1991. Climatic conditions of outflow ablative waters from Werenskiold Glacier in Spitsbergen. 1st International Symposium of Glacier Caves and Karst in Polar Regions. Proceedings, Madrid: 135-150.

Peterson B.J., Holmes R.M., McClelland J.W., Vörösmarty C.J., Lammers R.B., Shiklomanov A.I., Shiklomanov I.A., Rahmstorf S., 2002. Increasing river discharge to the Arctic Ocean. Science 298: 2171-2173. DOI: 10.1126/science. 1077445

Pękala K., 1987. Rzeźba i utwory czwartorzędowe przedpola lodowców Scotta i Renarda (Spitsbergen) (Relief and Quaternary deposits on the Scott and Renard glaciers forefield (Spitsbergen). In: Repelewska-Pękalowa J., Harasimiuk M., Pękala K. (eds), XIV Sympozjum Polarne. Wydawnictwo UMCS, Lublin: 84-87.

Piasecki J., Pulina M., 1975. Przepływ rzeki lodowcowej Werenskiolda i rzeki Brattegg jako funkcja niektórych parametrów klimatycznych w 1972 r. In: Polskie wyprawy na Spitsbergen 1972 i 1973 r. Materiaty z Sympozjum Spitsbergeńskiego. Wydawnictwo Uniwersytetu Wrocławskiego, Wrocław: 67-72.

Pillewizer W., 1939. Die kartographischen und gletscherkundlichen Ergebnisse der deutschen Spitzbergen-expedition 1938. Ergänzungsheft Nr. 238 zu Petermanns Geographische Mitteilungen. Justus Perthes, Gotha: 46.

Rachlewicz G., 2007. Mechanizmy wezbrań rzecznych w zlewniach zlodowaconych (przykłady z doliny Ebba, Spitsbergen Środkowy) [Mechanisms of river flooding in glaciated catchments (examples from Ebba Valley, Central Spitsbergen)]. In: Kostrzewski A., Szpikowski J. (eds), Funkcjonowanie geoekosystemów rzecznych. Vol. 4. Bogucki Wydawnictwo Naukowe, Poznań: 197-207.

Sobota I., 1998. Ablacja i odpływ z lodowca Waldemara w sezonie letnim 1997 (Ablation and outflow from Waldemar glacier in the 1997 summer). In: Repelewska-Pękalowa
J., Pękala K. (eds), Wyprawy Geograficzne na Spitsbergen 1998. Wydawnictwo UMSC, Lublin: 149-168.

Sobota I., 2004. Wpływ warunków pogodowych na zmienność ablacji lodowca Waldemara i lodowca Ireny w sezonie letnim 2003 roku (Influence of meteorological conditions on ablation of Waldemar Glacier and Irene Glacier (NW Spitsbergen) in the Summer season of 2003). Problemy Klimatologii Polarnej 14: 95-106.

Sobota I., 2011. Snow accumulation, melt, mass loss, and the near-surface ice temperature structure of Irenebreen. Svalbard Polar Science 5(3): 321-336. DOI: 10.1016/j.polar.2011.06.003

Sobota I., 2013. Wspótczesne zmiany kriosfery pótnocno-zachodniego Spitsbergenu na przykładzie regionu Kaffioyry (Contemporary changes of the cryosphere of north-western Spitsbergen based on the example of the Kaffioyra region). Wydawnictwo Naukowe UMK, Torun.

Sobota I., 2014. Changes in dynamics and runoff from the High Arctic glacial catchment of Waldemarbreen, Svalbard. Geomorphology 212: 16-27. DOI: 10.1016/j.geomorph.2013.04.001.

Sobota I., Nowak M., 2012. Dynamika odpływu ze zlodowaconej zlewni Rzeki Waldemara (NW Spitsbergen) w sezonach letnich 2009-2011 (Dynamics of the outflow from the glacierized Waldemar river catchment (NW Spitsbergen) in summer seasons 2009-2011). Problemy Klimatologii Polarnej 22: 57-68.

Stenborg T., 1965. Problems concerning winter run-off from glaciers. Geografiska Annaler Series A, Physical Geography 47: 141-184.

Stenborg T., 1968. Glacier drainage connected with ice structures. Geografiska Annaler Series A, Physical Geography 50: 25-53.

Sund M., 2008. Polar hydrology. Norwegian Water Resources and Energy Directorate's work in Svalbard. NVE Report 2. Norwegian Water Resources and Energy Directorate, Oslo.

Syvitski J.P.M., 2002. Sediment discharge variability in Arctic rivers: implications for a warmer future. Polar Research 21(2): 323-330. DOI: 10.1111/j.1751 8369.2002.tb00087.x.

Szczepanik W., Marciniak K., 1989. Wpływ ekstremalnych warunków pogodowych na zjawiska hydrologiczne na przykładzie wybranych rzek z regionu Kaffioyry (NW Spitsbergen) (Influence of extremal weather conditions on hydrological phenomena). In: Olszewski A. (ed.), XVI Sympozjum Polarne. Wydawnictwo UMK, Toruń: 185-188.

Tranter M., Brown G.H., Hodson A.J., Gurnell A.M., 1996. Hydrochemistry as an indicator of subglacial drainage system structure: a comparison of Alpine and sub-polar environments. Hydrological Processes 10(4): 541-556. DOI: 10.1002/(sici)1099-1085(199604)10:4<541:aid-hyp391>3.3.co;2-9. 